\title{
Associations between sequence variations in the mitochondrial DNA D-loop region and outcome of hepatocellular carcinoma
}

\author{
SHILAI LI, PEIQI WAN, TAO PENG, KAIYIN XIAO, MING SU, LIMING SHANG, BANGHAO XU, \\ ZHIXIONG SU, XINPING YE, NING PENG, QUANLIN QIN and LEQUN LI \\ Department of Hepatobiliary Surgery, The First Affiliated Hospital of Guangxi Medical University, \\ Nanning, Guangxi 530021, P.R. China
}

Received January 27, 2015; Accepted February 24, 2016

DOI: $10.3892 / \mathrm{ol} .2016 .4466$

\begin{abstract}
The association between mitochondrial DNA (mtDNA) polymorphisms or mutations and the prognoses of cancer have been investigated previously, but the results have been ambiguous. In the present study, the associations between sequence variations in the mtDNA D-loop region and the outcomes of patients with hepatocellular carcinoma (HCC) were analysed. A total of 140 patients with HCC (123 males and 17 females), who were hospitalised to undergo radical resection, were studied. Polymerase chain reaction and direct sequencing were performed to detect the sequence variations in the mtDNA D-loop region. Multivariate and univariate analyses were conducted to determine important factors in the prognosis of HCC. A total of 150 point sequence variations were observed in the 140 cases (13 point mutations, 8 insertions, 20 deletions and 116 polymorphisms). The variation rate was $13.4 \%(150 / 1,122)$. mtDNA nucleotide $150(\mathrm{C} / \mathrm{T})$ was an independent factor in the logistic regression for early/late recurrence of HCC. Patients with 150T appeared to have later recurrences. In a Cox proportional hazards regression model, hepatitis B virus DNA, Child-Pugh class, differentiation degree, tumour-node-metastasis (TNM) stage, nucleotide 16263 (T/C) and nucleotide 315 (N/insertion C) were independent factors for tumour-free survival time. Patients with the $16263 \mathrm{~T}$ allele had a greater tumour-free survival time than patients with the 16263C allele. Similarly, patients with 315 insertion $C$ had a superior tumour-free survival time when compared with patients with $315 \mathrm{~N}$ (normal). In the Cox proportional hazards regression model, recurrence type (early/late), Child-Pugh class, TNM stage and adjuvant treatment after tumour recurrence (none or one/more than one treatment) were independent
\end{abstract}

Correspondence to: Professor Lequn Li, Department of Hepatobiliary Surgery, The First Affiliated Hospital of Guangxi Medical University, 6 Shuangyong Road, Nanning, Guangxi 530021, P.R. China

E-mail: lilequn2011@aliyun.com

Key words: mitochondrial DNA, polymorphism, hepatocellular carcinoma, outcome factors for overall survival. None of the mtDNA variations served as independent factors. Patients with late recurrence, Child-Pugh class A, and low TNM stages and/or those who received more than one adjuvant treatment following tumour recurrence had favourable outcomes. mtDNA D-loop polymorphisms were associated with early recurrence and tumour-free survival time, but not with overall survival. mtDNA D-loop mutations in $\mathrm{HCC}$ were infrequent and lacked prognostic utility. The detection of mtDNA D-loop polymorphisms may assist in identifying risk factors for HCC prognosis, particularly for the short-term outcome, thereby aiding the construction of an appropriate therapeutic strategy.

\section{Introduction}

Hepatocellular carcinoma (HCC) is one of the most prevalent forms of cancer worldwide, and is associated with chronic liver diseases, including hepatitis $\mathrm{B}$ and/or hepatitis $\mathrm{C}$ viral infection $(1,2)$. The overall survival of HCC patients is poor due to the high recurrence rate following hepatic resection (3). Chronic viral inflammation and reactive oxygen species (ROS), which are increased by chronic inflammation, are considered as the most important factors in HCC carcinogenesis (4).

Mitochondria are present in eukaryotic cells and are ubiquitous organelles that generate energy in the form of ATP through the process of oxidative phosphorylation (OXPHOS) (5). The human mitochondrial genome is 16,569 bp in length, with double-stranded circular DNA molecules containing 37 genes encoding 13 peptides for the OXPHOS apparatus, in addition to 22 transfer RNAs and 2 ribosomal RNAs, which are required for protein synthesis in the mitochondria. Furthermore, mitochondrial DNA (mtDNA) contains a non-coding region that accommodates a unique displacement loop (D-loop), which controls the transcription and replication of mtDNA. mtDNA is considered to be more vulnerable to oxidative damage and to have a higher mutation rate compared with nuclear DNA due to the lack of a repair system and protective histones within the mitochondria $(5,6)$. A number of prevalent polymorphisms have been identified in mtDNA, and the majority accumulate in the D-loop (or regulatory) region (7). Damage to and somatic mutations of mtDNA may lead to impairment of the OXPHOS system and increased ROS production, which subsequently enhances the rate of 
DNA mutations (8). Numerous researchers have reported that mtDNA mutations or polymorphisms, particularly in the D-loop region, were detected in different types of cancer, including human colorectal (9), ovarian (10) and thyroid (11) cancer, in addition to HCC (12). Furthermore, the association between mtDNA polymorphisms or mutations and the prognoses of cancer has also been investigated, but the results have been ambiguous. In the present study, the polymorphisms and mutations in the mtDNA D-loop region of patients with HCC were analysed, and the clinical significance of these variations was investigated.

\section{Materials and methods}

Patients and tissue specimens. A total of 140 patients with HCC who were hospitalised for radical resection in the Department of Hepatobiliary Surgery, The First Affiliated Hospital of Guangxi Medical University (Nanning, China) from January 2003 to August 2012 were studied. Patients who died during the perioperative period or those who exhibited recurrence within 2 months post-surgery were excluded. All of the tumour tissues were confirmed as HCC by histopathology. Of the 140 patients, 123 were male and 17 were female and the median age was 47 years (range, 25-79 years). All tissues were placed and stored in liquid nitrogen immediately after surgical resection, according to the guidelines of the Human Tissue Research Committee of The First Affiliated Hospital of Guangxi Medical University. Written informed consent was obtained from each participant prior to enrolment, and this study was approved by the Medical Ethics Committee of The First Affiliated Hospital of Guangxi Medical University.

mtDNA extraction and D-loop amplification. All tumour tissues were removed from liquid nitrogen and refrigerated at $-80^{\circ} \mathrm{C}$ until DNA extraction. Total cellular DNA was extracted from the tumour tissue according to the specifications of the TIANamp Genomic DNA kit (Tiangen Biotech Co., Ltd., Beijing, China), and was stored at $-20^{\circ} \mathrm{C}$ until mtDNA amplification. The mtDNA fragments were amplified using the following primers: Forward, 5'-ATTCTAACCTGA ATCGGAGG-3'; and reverse, 5'-GATGCTTGCATGTGTAAT CT-3' (Sangon Biotech Co., Ltd., Shanghai, China). The fragments were $1,528 \mathrm{bp}$ in length, including the complete D-loop region of 1,122 bp, as described previously (13). Each DNA sample (50 ng) was amplified using polymerase chain reaction (PCR; MJ Research PTC-200 Thermal Cycler; Bio-Rad Laboratories, Inc., Hercules, CA, USA). PCR was performed in a final volume of $50 \mu \mathrm{l}$ with the Taq ${ }^{\mathrm{TM}}$ Hot-Start Version PCR kit (Takara Bio, Inc., Otsu, Japan). The thermal profile was as follows: Initial incubation at $95^{\circ} \mathrm{C}$ for $5 \mathrm{~min}$, followed by 35 cycles of $95^{\circ} \mathrm{C}$ for $1 \mathrm{~min}, 55^{\circ} \mathrm{C}$ for $1 \mathrm{~min}$ and $72^{\circ} \mathrm{C}$ for $2 \mathrm{~min}$, and a final extension at $72^{\circ} \mathrm{C}$ for $7 \mathrm{~min}$.

Direct sequencing and analysis of the D-loop region of $m t D N A$. Aberrant PCR products were purified with a PCR purification kit (Beijing Sunbiotech Co., Ltd., Beijing, China) and were sequenced with an 3730xl DNA Analyzer (Applied Biosystems; Thermo Fisher Scientific, Inc., Waltham, MA, USA). The direct sequencing primers were the same as those used for amplification. As there were numerous structures containing poly-C in the mtDNA D-loop region, the following two additional primers were used for PCR experiments (F2: 5'-AATACTTGACCACCTGTAG-'3; and F3: 5-'CCT ATGTCGCAGTATCTGTC-'3) to sequence the entire mtDNA D-loop region (13). Multiple primers overlapped the sequenced regions. All of the variations were confirmed by repeated analysis of the mtDNA extracted from the tissue samples. All of these procedures were performed by Beijing Sunbiotech Co., Ltd. (Beijing, China).

The sequences of the tumour tissues were compared with the revised Cambridge Reference Sequence (GenBank ${ }^{\circledR}$ NC_012920) using DNASTAR Lasergene software version 7.1 (DNASTAR, Inc., Madison, WI, USA). Subsequently, the variations in the sequences were considered polymorphisms or mutations according to the mtDNA database (www.mitomap.org).

Follow-up. The patients with HCC were recommended to receive postoperative adjuvant transcatheter hepatic arterial chemoembolisation (TACE). All patients were followed up in the outpatient clinic every 3 months, and their serum $\alpha$-fetoprotein levels were measured. Hepatic ultrasonography was performed every 2-4 months from the date of initial treatment until December 31, 2014, or mortality. If any signs of recurrence were suspected, further investigation was performed using abdominal computed tomography or magnetic resonance imaging to confirm the diagnosis. If recurrence was verified, further treatment was recommended according to the condition of the patient and included re-operation, TACE, percutaneous ethanol injection (PEI) and/or radiofrequency ablation (RFA).

Statistical analysis. SPSS software version 13.0 (SPSS, Inc., Chicago, IL, USA) was used to perform the analyses. For the univariate analysis, the $\chi^{2}$ test and Kaplan-Meier method (compared using the log-rank test) were employed. For the multivariate analysis, a stepwise logistic regression model and the Cox proportional hazards regression model were used to identify the most important factors. $\mathrm{P}<0.05$ was considered to indicate a statistically significant difference.

\section{Results}

Patient follow-up. The median follow-up time was 40.9 months (range, $8-126$ months). A total of 100 patients with HCC received postoperative adjuvant TACE prior to signs of recurrence, while the remaining 40 patients did not receive any TACE treatment and only attended follow-up visits at the outpatient clinic. As of December 31, 2014, there were 129 cases with recurrence (median time, 12 months; range, 3-77 months) and 107 mortalities (median time, 37 months; range, 8-126 months). Of the 129 patients with recurrence, surgery was performed in 42 cases (49 times altogether, twice for 7 cases); additionally, in the same group of patients, TACE was performed 354 times, RFA was performed 31 times and PEI was performed 152 times. A total of 73 patients received more than one treatment following recurrence.

Variations in the mtDNA D-loop region. A total of 150 point sequence variations were observed among the 140 cases. 
Table I. Clinicopathological factors for early recurrent hepatocellular carcinoma in a stepwise logistic regression model.

\begin{tabular}{lcccrr}
\hline Clinicopathological variables & B & SE & Exp (B) & $95.0 \%$ CI for Exp (B) & P-value \\
\hline HBV DNA & 1.152 & 0.446 & 3.164 & $1.320-7.582$ & 0.010 \\
Pathological differentiation degree & 0.841 & 0.402 & 2.318 & $1.054-5.100$ & 0.037 \\
TNM & 1.033 & 0.230 & 2.809 & $1.791-4.405$ & $<0.001$ \\
Nucleotide 150 (C/T) & -1.512 & 0.491 & 0.220 & $0.084-0.577$ & 0.002
\end{tabular}

Variables with $\mathrm{P} \leq 0.1$ in the univariate analysis were entered in the multivariate analysis. A stepwise logistic regression model (forward likelihood ratio) was used with the standard $\mathrm{P}$-value $\leq 0.05$ entered into the equation, while variables with a $\mathrm{P}$-value of $>0.1$ were removed. SE, standard error; CI, confidence interval; HBV DNA, hepatitis B virus DNA; TNM, tumour-node-metastasis; C, cytosine; T, thymine; B, partial regression coefficient; Exp (B), exponentiation of the B coefficient (an odds ratio).

Of these variations, there were 13 point mutations, 8 insertions, 20 deletions and 116 polymorphisms (including a few insertions and deletions according to the mtDNA database). The variation rate was $13.4 \%(150 / 1,122)$. The majority of the variations were located in hypervariable segments I (NT57-372) and II (NT16024-16383), and they consisted of $46(30.7 \%, 46 / 150)$ and 63 points $(42.0 \%, 63 / 150)$, respectively. No large-scale deletions were observed.

Clinicopathological characteristics in patients with early and late recurrence. Early recurrence was characterised by a recurrence time of $<1$ year post-radical resection, while recurrences that occurred after 1 year were characterised as late recurrences. In the univariate analysis, the clinicopathological characteristics [including age, tumour size, tumour capsule, surgical margin, hepatitis B e antigen, hepatitis B virus DNA (HBV DNA), differentiation degree (Edmondson-Steiner grade), cirrhosis, microscope embolus, TNM stage, nucleotide 16129 (G/A), nucleotide 16192 (C/T), nucleotide 16297 (T/C), nucleotide 150 (C/T), nucleotide 199 (T/C) and nucleotide 204 (T/C) of mtDNA] were significantly different between the early and late recurrence groups $(\mathrm{P}<0.05)$. However, only HBV DNA, differentiation degree, TNM stage and nucleotide $150(\mathrm{C} / \mathrm{T})$ of mtDNA were independent factors in the logistic regression. Nucleotide $150(\mathrm{C} / \mathrm{T})$ of mtDNA was a protective factor, while the remainder were risk factors. Patients with the $150 \mathrm{~T}$ allele appeared to exhibit later recurrence (Table I).

Associations between HCC prognosis and clinicopathological characteristics. Patients with HCC were divided into different groups based on their clinicopathological characteristics and genotypes at each variation site. The postoperative tumour-free and overall survival curves were subsequently plotted using the Kaplan-Meier method and were compared by the log-rank test. The Cox proportional hazards regression model was also used to identify the most important factors.

Among the 140 cases, the median tumour-free survival time was 12 months, and the recurrence rates for 1,2,3,4 and 5 years were $50,70,79,89$ and $92 \%$, respectively. In the univariate analysis, the clinicopathological characteristics, including tumour size, tumour capsule, HBV DNA, Child-Pugh class, differentiation degree, tumour necrosis, microscopic embolism, TNM stage, nucleotide 16129 (G/A), nucleotide 16263
(T/C), nucleotide 310 (T/CTC/other types) and nucleotide 315 (N/insertion C) were significantly different between the groups. No significant differences were observed between the mutation and normal groups, while in the Cox proportional hazards regression model, only HBV DNA, Child-Pugh class, differentiation degree, TNM stage, nucleotide 16263 (T/C) and nucleotide 315 (N/insertion C) were independent factors for tumour-free survival time. Regarding the mtDNA variations, patients with the $16263 \mathrm{~T}$ allele had a greater tumour-free survival time than patients with the $16263 \mathrm{C}$ allele. Similarly, patients with 315 insertion $\mathrm{C}$ had a superior tumour-free survival time when compared with patients with the $315 \mathrm{~N}$ genotype (normal) (Table II; Fig. 1 and 2).

Among the 140 cases, the median overall survival was 37 months, and the survival rates for 1, 2, 3, 4 and 5 years were $94,72,56,40$ and $27 \%$, respectively. In the univariate analysis, the clinicopathological characteristics, including recurrence type (early/late), age, tumour size, tumour number, HBV DNA, Child-Pugh class, differentiation degree, tumour necrosis, microscopic embolism, TNM stage, adjuvant treatment following tumour recurrence (none or one/more than one treatment), nucleotide 16263 (T/C), nucleotide $150(\mathrm{C} / \mathrm{T})$, nucleotide 249 (insertion A/N), nucleotide 310 (T/CTC/other types), nucleotide 315 (N/insertion $\mathrm{C}$ ) and mutations were all independent factors for overall survival, while in the Cox proportional hazards regression model, only recurrence type (early/late), Child-Pugh class, TNM stage and adjuvant treatment following tumour recurrence (none or one/more than one treatment) were independent factors for overall survival. None of the mtDNA variations were independent factors. Patients with late recurrence, Child-Pugh class A, and a low TNM stage and/or those who received more than one adjuvant treatment following tumour recurrence had favourable outcomes (Table III).

\section{Discussion}

Unlike other forms of cancer, hepatocellular carcinoma is consistently associated with chronic liver diseases $(14,15)$. The majority of studies regarding $\mathrm{HCC}$ have focused on the cellular nucleus, with a number of variations in genes and their expression identified as potential carcinogenesis factors $(16,17)$. However, the true cause of HCC carcinogenesis is yet to be elucidated. In population studies for the detection of a variety 
Table II. Multivariate analysis of the clinicopathological factors using Cox's proportional hazards regression model for tumour-free survival.

\begin{tabular}{lccccr}
\hline Clinicopathological variables & B & SE & Exp (B) & 95.0\% CI for Exp (B) & P-value \\
\hline HBV DNA & 0.662 & 0.190 & 1.939 & $1.337-2.814$ & $<0.001$ \\
Child-Pugh class & -0.591 & 0.198 & 0.554 & $0.375-0.817$ & 0.003 \\
Pathological differentiation degree & -0.425 & 0.211 & 0.654 & $0.432-0.989$ & 0.044 \\
TNM & 0.517 & 0.093 & 1.677 & $1.399-2.011$ & $<0.001$ \\
Nucleotide 16263 (T/C) & 0.708 & 0.315 & 2.030 & $1.095-3.762$ & 0.025 \\
Nucleotide 315 insertion C & -0.462 & 0.186 & 0.630 & $0.437-0.908$ & 0.013 \\
\hline
\end{tabular}

Variables with $\mathrm{P} \leq 0.1$ in the univariate analysis entered the multivariate analysis. A stepwise Cox proportional hazards regression model (forward likelihood ratio) was used with the standard $\mathrm{P}$-value $\leq 0.05$ entered into the equation, while variables with a $\mathrm{P}$-value $>0.1$ were removed. SE, standard error; CI, confidence interval; HBV DNA, hepatitis B virus DNA; TNM, tumour-node-metastasis; T, thymine; C, cytosine; B, partial regression coefficient; Exp (B), exponentiation of the B coefficient (an odds ratio).

Table III. Multivariate analysis of the clinicopathological factors using Cox's proportional hazards regression model for overall survival.

\begin{tabular}{lrrrr}
\hline Clinicopathological variables & B & SE & Exp (B) & 95.0\% CI for Exp (B) \\
\hline Recurrence type (early/late) & 2.017 & 0.271 & 7.513 & $4.413-12.791$ \\
Child Pugh class & -0.669 & 0.206 & 0.512 & $0.342-0.766$ \\
TNM & 0.226 & 0.110 & 1.253 & $1.009-1.555$ \\
Adjuvant treatment post-tumour recurrence & -0.431 & 0.207 & 0.650 & $0.433-0.976$ \\
(none or one/more than one treatment) & & & & 0.041 \\
\hline
\end{tabular}

Variables with $\mathrm{P} \leq 0.1$ in the univariate analysis entered the multivariate analysis. A stepwise Cox proportional hazards regression model (forward likelihood ratio) was used with the standard $\mathrm{P}$-value $\leq 0.05$ entered into the equation, while variables with a $\mathrm{P}$-value $>0.1$ were removed. SE, standard error; CI, confidence interval; TNM, tumour-node-metastasis; B, partial regression coefficient; Exp (B), exponentiation of the B coefficient (an odds ratio).

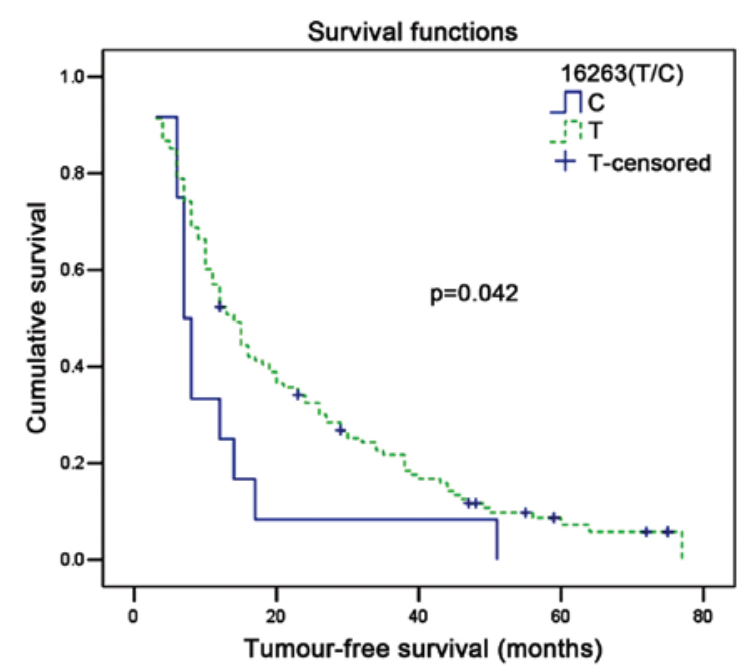

Figure 1. Tumour-free survival curve of patients with HCC with the mtDNA 16263 allele $(\mathrm{T} / \mathrm{C})$. The median tumour-free survival time was 14 and 7 months for $16263 \mathrm{~T}$ and $16263 \mathrm{C}$, respectively.

of diseases and tumours, polymorphisms of or alterations in mtDNA have emerged as novel biomarkers (5). A number of mutations or polymorphisms in the coding and non-coding

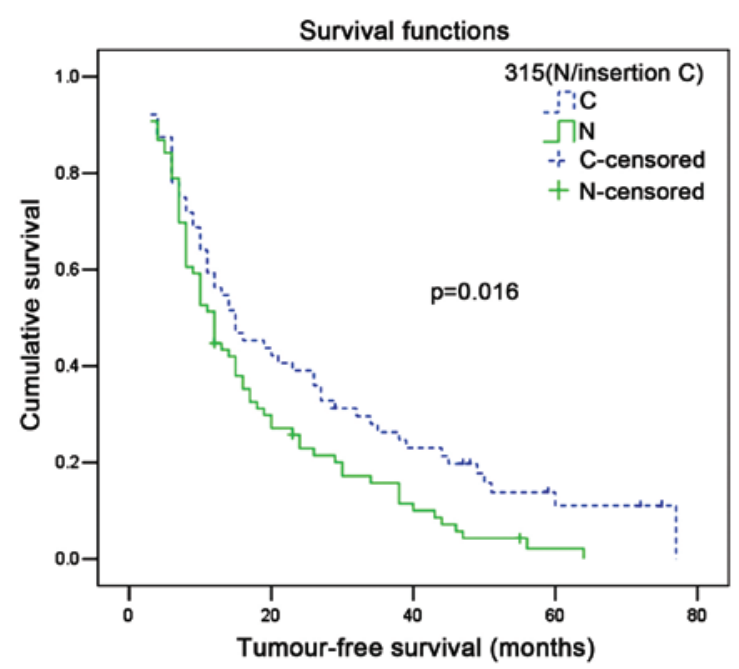

Figure 2. Tumour-free survival curve of patients with HCC with the mtDNA 315 allele (normal/insertion $\mathrm{C}$ ). The median tumour-free survival time was 12 and 15 months for $315 \mathrm{~N}$ and $315 \mathrm{C}$, respectively.

regions of the mitochondrial genome have been associated with an elevated risk of cancer $(6,8)$. A few studies have reported that mtDNA mutations were correlated with tumorigenesis $(11,18)$, 
while others have focussed on their association with cancer and aetiology $(19,20)$. The detection of specific mutations in individual cancer populations has recently become a popular area of research. In a previous study conducted by the authors, it was observed that analysing sequence variations in the mtDNA D-loop region may determine useful biomarkers for identifying the cell clonal origin of synchronous multinodular HCC (13). In the current study, HCC tissue samples were screened for mtDNA polymorphisms and mutations, and their association with HCC prognoses were analysed.

The results demonstrated that mtDNA mutations are not ubiquitous in HCC, as only a small proportion (13/140, 9.3\%) of the HCC tissue samples had D-loop mutations. Previous studies have reported that mtDNA mutations were universal in cancer and useful for predicting the outcomes of patients (21-23). Yin et al (21) reported that $22 \%$ of HCC tissues studied carried a somatic mutation in the mtDNA D-loop region. Lee et al (22) examined mutations in the D-loop region of mtDNA in $61 \mathrm{HCC}$ specimens, and the results demonstrated that $39.3 \%$ carried somatic mutation(s) in the mtDNA D-loop. Furthermore, Wheelhouse et al (23) reported that the percentage of tumour tissue from patients with HCC harbouring D-loop mutations was $59 \%$. However, in other studies, mtDNA mutations were not ubiquitous, which is comparable to the present study (24). In head and neck squamous cell carcinoma (HNSCC), pooled data from nine studies investigating D-loop mutations in $>400$ cases of HNSCC indicated that approximately one-third of the HNSCC cases harboured D-loop mutations; however, there was significant variation between different studies (range, 2-67\%) (24). Explanations for these differences may be include variation in the methods of mutation detection or the intrinsic genetic heterogeneity of cancer.

In the present study, there were no significant associations observed between mtDNA mutations and the prognosis of patients with HCC. mtDNA mutations in the HCC tissues appeared to be infrequent and lack prognostic utility, consistent with a previous study (7). However, Liu et al (25) observed that patients with oral squamous cell carcinoma with D-loop mutations had superior survival rates when compared with patients without such mutations (2-year disease-specific survival rate: 73.4 vs. $45.0 \%$, respectively). In a breast cancer study, Kuo et al (26) reported that somatic mutations in the mtDNA D-loop and in tumour protein 53 (TP53) were independent of each other, indicating that the number of somatic mtDNA D-loop region mutations may be an indicator of poor prognosis through a TP53-independent mechanism. However, Challen et al (24) demonstrated that mtDNA mutations were not ubiquitous in HNSCC, and that there were no significant associations between D-loop mutations and determinants of clinical outcomes. The results were not consistent between studies, and according to our data, we hypothesised that the mutations in the mtDNA D-loop region were stochastic events that may not significantly affect the biology of HCC.

Sequence variations or polymorphisms in the mtDNA D-loop region are common in HCC. The majority of variations in the present study were located in hypervariable segments I (NT57-372) and II (NT16024-16383) (30.7 and 42.0\%, respectively). Of these variations, the nucleotide $150(\mathrm{C} / \mathrm{T})$ polymorphism of mtDNA was associated with HCC recurrence. The $150 \mathrm{~T}$ allele frequency was much higher in late recurrence of HCC. A number of studies have reported that the $150(\mathrm{C} / \mathrm{T})$ polymorphism was correlated with longevity (27-29). It is considered that the $150(\mathrm{C} / \mathrm{T})$ transition functions in remodelling mtDNA replication, thus, if the $150(\mathrm{C} / \mathrm{T})$ transition is present, replication of the mtDNA heavy strand would begin at position $149 \mathrm{~T}$ instead of position $151 \mathrm{C}$, which would subsequently affect ATP synthesis through the process of OXPHOS in cell metabolism (30). This result was similar to that of a previous study of cervical cancer, in which Zhai et al (31) reported that a mitochondrial $150(\mathrm{C} / \mathrm{T})$ polymorphism increased the risk of cervical cancer and HPV infection. However, based on the results of the present study, it was not possible to determine the mechanism of or provide further details regarding this association. In addition, the current study demonstrated that the $150(\mathrm{C} / \mathrm{T})$ polymorphism was not associated with tumour-free or overall survival. Further research investigating the role of nucleotide $150(\mathrm{C} / \mathrm{T})$ is required.

In the present study, two polymorphisms, 16263 (T/C) and insertion $315 \mathrm{C}$, were identified as independent predictive factors for tumour-free survival time. Patients with the $16263 \mathrm{~T}$ allele had a greater tumour-free survival time than patients with the $16263 \mathrm{C}$ allele. Similarly, patients with 315 insertion C had a superior tumour-free survival time when compared with patients with $315 \mathrm{~N}$ (normal). However, none of the mtDNA variations were identified as independent predictors of overall survival. The association between mtDNA polymorphisms and prognosis has been analysed in various types of cancer $(7,32,33)$. Wang et al (7) investigated the predictive power of D-loop single nucleotide polymorphisms (SNPs) in patients with $\mathrm{HCC}$, and two SNP sites (nucleotides $150 \mathrm{C} / \mathrm{T}$ and $146 \mathrm{~T} / \mathrm{C}$ ) were identified by the log-rank test as statistically significant predictors of HCC survival. Furthermore, in a multivariate analysis, allele 146 was identified as an independent predictor, and the survival time of patients with allele $146 \mathrm{C}$ was significantly less than that of patients with allele 146T. Ding et al (32) also demonstrated that allele 16390 was an independent predictor of non-small cell lung cancer outcome. The survival time of patients with allele 16390A was significantly shorter than that of patients with allele 16390G. Guo et al (33) identified that nucleotide $16266 \mathrm{C} / \mathrm{T}$ was associated with age at onset in patients with oesophageal squamous cell carcinoma. The age at onset of patients with the minor allele $\mathrm{T}$ genotype was significantly younger than that of patients with the $\mathrm{C}$ genotype at the 16266 site. However, based on the data from the present study, it was not possible to determine a specific variation in patients with HCC. Further investigation is required to determine whether these conclusions may be extended to other patients with HCC.

Although the mechanisms of mtDNA variations in tumour development and progression have not yet been elucidated, studies suggest that variations in the mtDNA D-loop region may induce significant alterations in mitochondrial function. The mtDNA D-loop is crucial for regulating the replication and expression of the mitochondrial genome. It was previously reported that mtDNA variations had subtle effects on the electron transport chain (34). However, the accumulation of numerous subtle changes may result in significant consequences. Furthermore, mitochondrial respiratory chain activity was affected notably, which is responsible for the 
high ROS release and nuclear genome damage, in addition to cancer initiation and promotion $(5,6,8)$. High levels of ROS have been demonstrated to be significant in activating cell apoptosis and infliction of injury to the genome $(8,35)$. Thus, the present study hypothesised that variations in the mtDNA D-loop region may affect the entire function of mtDNA, further interfering with nuclear genome expression and function. However, the questions as to whether variations in the mtDNA D-loop region are the result or cause of carcinogenesis requires further investigation.

In conclusion, the results of the current study demonstrated that mtDNA D-loop polymorphisms were associated with early recurrence and tumour-free survival time, but not with overall survival. mtDNA D-loop mutations in patients with HCC were infrequent and lacked prognostic utility. Therefore, the detection of mtDNA D-loop polymorphisms may aid in identifying risk factors for HCC prognosis, particularly for the short-term outcome, and thus aiding the construction of an appropriate therapeutic strategy. However, mtDNA D-loop variations lacked utility in predicting long-term outcomes, therefore further biomarkers require investigation.

\section{Acknowledgements}

The present study was supported by a grant from the National Natural Science Foundation of China (no. 81160262). The funding body served no role in the study design, data collection and analysis, decision to publish or manuscript preparation. The language, grammar, punctuation and spelling of the original manuscript was edited by the American Journal Experts.

\section{References}

1. Bray F, Ren JS, Masuyer E and Ferlay J: Global estimates of cancer prevalence for 27 sites in the adult population in 2008 . Int J Cancer 132: 1133-1145, 2013.

2. Jemal A, Bray F, Center MM, Ferlay J, Ward E and Forman D: Global cancer statistics. CA Cancer J Clin 61: 69-90, 2011.

3. Portolani N, Coniglio A, Ghidoni S, et al: Early and late recurrence after liver resection for hepatocellular carcinoma: Prognostic and therapeutic implications. Ann Surg 243: 229-235, 2006.

4. Lim W, Kwon SH, Cho H, et al: HBx targeting to mitochondria and ROS generation are necessary but insufficient for HBV-induced cyclooxygenase-2 expression. J Mol Med Berl 88: 359-369, 2010.

5. Zapico SC and Ubelaker DH: mtDNA mutations and their role in aging, diseases and forensic sciences. Aging Dis 4: 364-380, 2013

6. Singh AK, Pandey P, Tewari M, Pandey HP and Shukla HS: Human mitochondrial genome flaws and risk of cancer. Mitochondrial DNA 25: 329-334, 2014.

7. Wang C, Zhang F, Fan H, et al: Sequence polymorphisms of mitochondrial D-loop and hepatocellular carcinoma outcome. Biochem Biophys Res Commun 406: 493-496, 2011.

8. Fogg VC, Lanning NJ and Mackeigan JP: Mitochondria in cancer: At the crossroads of life and death. Chin J Cancer 30: 526-539, 2011

9. Akouchekian M, Houshmand M, Hemati S, et al: High rate of mutation in mitochondrial DNA displacement loop region in human colorectal cancer. Dis Colon Rectum 52: 526-530, 2009.

10. Liu VW, Shi HH, Cheung AN, et al: High incidence of somatic mitochondrial DNA mutations in human ovarian carcinomas. Cancer Res 61: 5998-6001, 2001.

11. Ding Z, Ji J, Chen G, et al: Analysis of mitochondrial DNA mutations in D-loop region in thyroid lesions. Biochim Biophys Acta 1800: 271-274, 2010.

12. Yin PH, Wu CC, Lin JC, et al: Somatic mutations of mitochondrial genome in hepatocellular carcinoma. Mitochondrion 10: 174-182, 2010.

13. Li SL, Su M, Peng T, et al: Clinicopathologic characteristics and prognoses for multicentric occurrence and intrahepatic metastasis in synchronous multinodular hepatocellular carcinoma patients. Asian Pac J Cancer Prev 14: 217-223, 2013.
14. Weinmann A, Koch S, Niederle IM, et al: Trends in epidemiology, treatment and survival of hepatocellular carcinoma patients between 1998 and 2009: An analysis of 1066 cases of a German HCC Registry. J Clin Gastroenterol 48: 279-289, 2014.

15. Nault JC: Pathogenesis of hepatocellular carcinoma according to aetiology. Best Pract Res Clin Gastroenterol 28: 937-947, 2014.

16. Kirstein MM and Vogel A: The pathogenesis of hepatocellular carcinoma. Dig Dis 32: 545-553, 2014.

17. Liu M, Jiang L and Guan XY: The genetic and epigenetic alterations in human hepatocellular carcinoma: A recent update. Protein Cell 5: 673-691, 2014.

18. Nishikawa M, Nishiguchi S, Shiomi S, et al: Somatic mutation of mitochondrial DNA in cancerous and noncancerous liver tissue in individuals with hepatocellular carcinoma. Cancer Res 61: 1843-1845, 2001

19. Zhang R, Zhang F, Wang C, Wang S, Shiao YH and Guo Z: Identification of sequence polymorphism in the D-Loop region of mitochondrial DNA as a risk factor for hepatocellular carcinoma with distinct etiology. J Exp Clin Cancer Res 29: 130, 2010.

20. Sharma H, Singh A, Sharma C, Jain SK and Singh N: Mutations in the mitochondrial DNA D-loop region are frequent in cervical cancer. Cancer Cell Int 5: 34, 2005.

21. Yin PH, Lee HC, Chau GY, Wu YT, Li SH, Lui WY, Wei YH, Liu TY and Chi CW: Alteration of the copy number and deletion of mitochondrial DNA in human hepatocellular carcinoma. Br J Cancer 90: 2390-2396, 2004.

22. Lee HC, Li SH, Lin JC, Wu CC, Yeh DC and Wei YH: Somatic mutations in the D-loop and decrease in the copy number of mitochondrial DNA in human hepatocellular carcinoma. Mutat Res 547: 71-78, 2004.

23. Wheelhouse NM, Lai PB, Wigmore SJ, Ross JA and Harrison DJ: Mitochondrial D-loop mutations and deletion profiles of cancerous and noncancerous liver tissue in hepatitis B virus-infected liver. Br J Cancer 92: 1268-1272, 2005.

24. Challen C, Brown H, Cai C, Betts G, Paterson I, Sloan P, West C, Birch-Machin M and Robinson M: Mitochondrial DNA mutations in head and neck cancer are infrequent and lack prognostic utility. Br J Cancer 104: 1319-1324, 2011.

25. Liu SA, Jiang RS, Chen FJ, Wang WY and Lin JC: Somatic mutations in the D-loop of mitochondrial DNA in oral squamous cell carcinoma. Eur Arch Otorhinolaryngol 269: 1665-1670, 2012.

26. Kuo SJ, Chen M, Ma GC, Chen ST, Chang SP, Lin WY, Chen YC, Lee TH, Lin TT and Liu CS: Number of somatic mutations in the mitochondrial D-loop region indicates poor prognosis in breast cancer, independent of TP53 mutation. Cancer Genet Cytogenet 201: 94-101, 2010.

27. Niemi AK, Hervonen A, Hurme M, Karhunen PJ, Jylhä M and Majamaa K: Mitochondrial DNA polymorphisms associated with longevity in a Finnish population. Hum Genet 112: 29-33, 2003.

28. Niemi AK, Moilanen JS, Tanaka M, Hervonen A, Hurme M, Lehtimäki T, Arai Y, Hirose N and Majamaa K: A combination of three common inherited mitochondrial DNA polymorphisms promotes longevity in Finnish and Japanese subjects. Eur J Hum Genet 13: 166-170, 2005.

29. Ren WH, Li XH, Zhang HG, Deng FM, Liao WQ, Pang Y, Liu YH, Qiu MJ, Zhang GY and Zhang YG: Mitochondrial DNA haplogroups in a Chinese Uygur population and their potential association with longevity. Clin Exp Pharmacol Physiol 35: 1477-1481, 2008.

30. Zhang J, Asin-Cayuela J, Fish J, et al: Strikingly higher frequency in centenarians and twins of mtDNA mutation causing remodeling of replication origin in leukocytes. Proc Natl Acad Sci USA 100: 1116-1121, 2003.

31. Zhai K, Chang L, Zhang Q, Liu B and Wu Y: Mitochondrial C150T polymorphism increases the risk of cervical cancer and HPV infection. Mitochondrion 11: 559-563, 2011.

32. Ding C, Li R, Wang P, Fan H and Guo Z: Sequence polymorphisms of the mitochondrial displacement loop and outcome of non-small cell lung cancer. Exp Ther Med 3: 861-864, 2012.

33. Guo Z, Yang H, Zhang F, Zhang R and Wang C: Single nucleotide polymorphisms in the mitochondrial displacement loop and age-at-onset of esophageal squamous cell carcinoma. Oncol Lett 3: 482-484, 2012.

34. Polyak K, Li Y, Zhu H, Lengauer C, Willson JK, Markowitz SD, Trush MA, Kinzler KW and Vogelstein B: Somatic mutations of the mitochondrial genome in human colorectal tumours. Nat Genet 20: 291-293, 1998.

35. Zamzami N and Kroemer G: The mitochondrion in apoptosis: How Pandora's box opens. Nat Rev Mol Cell Biol 2: 67-71, 2001. 